Supporting Information for:

\title{
Topologically Diverse Human Membrane Proteins Partition to Liquid-Disordered Domains in Phase-Separated Lipid Vesicles
}

\author{
Jonathan P. Schlebach, ${ }^{1,2}$ Paul Barrett, ${ }^{1,2}$ Charles A. Day, ${ }^{3,4}$ Ji Hun Kim, ${ }^{1,2}$ \\ Anne Kenworthy, ${ }^{3,4^{*}}$ and Charles R. Sanders ${ }^{1,2 *}$ \\ Department of Biochemistry, ${ }^{1}$ Center for Structural Biology, ${ }^{2}$ Department of Molecular \\ Physiology and Biophysics, ${ }^{3}$ Department of Cell and Developmental Biology, ${ }^{4}$ Vanderbilt \\ University School of Medicine, Nashville, Tennessee 37232 USA
}

This file includes:

1. Supplementary Methods

2. Supplementary References

3. Supplementary Figures 1-3 


\section{Supplemental Methods}

Protein Purification and Fluorescent Labelling of C99, Cav3, PMP22, and ctxB

To facilitate fluorescent labeling of these proteins, cysteine residues were introduced into the C99 and Cav3 genes. C99 studies were carried out using a single-cysteine variant (F675C) of human C99. Cav3 studies were also carried out using a single-cysteine variant (V25C) of human Cav3, which was produced in the background of an otherwise cysteine-free construct described previously (1). PMP22 studies were carried out using the wild-type form of human PMP22, which contains four native cysteines. Mutant constructs were produced using Quickchange Mutagenesis. Alexafluor 488-labeled ctxB was purchased from Invitrogen (San Diego, CA).

C99 was expressed and purified as described previously (2), except the protein was exchanged into SDS micelles on column and eluted in $20 \mathrm{mM}$ Tris (pH 7.8) containing $200 \mathrm{mM}$ $\mathrm{NaCl}, 250 \mathrm{mM}$ imidazole, and 0.2\% SDS (w/v). The $\mathrm{pH}$ was then lowered to 6.5 with $\mathrm{HCl}$ prior to a 10-fold concentration of the sample using an Amicon 10 KDa MWCO centrifugational concentrator (Millipore, Billerica, MA). To reduce disulfide bonds, a 100 mM TCEP (pH 6.5) stock solution was then added to a final concentration of $5 \mathrm{mM}$ prior to tumbling the sample overnight at room temperature. Cav3 was expressed and purified as described previously (1), except the protein was exchanged into SDS on column and eluted in $250 \mathrm{mM}$ imidazole (pH 6.5) containing $2 \mathrm{mM}$ TCEP and $0.2 \% \mathrm{SDS}(\mathrm{w} / \mathrm{v})$. Wild-type PMP22 was expressed and purified as described previously (3). Protein stocks were flash-frozen and in liquid nitrogen and stored at $-30^{\circ} \mathrm{C}$ until use. 
To fluorescently label the proteins, concentrated protein stock solutions were diluted into $40 \mathrm{mM}$ HEPES (pH 7.5) containing $150 \mathrm{mM} \mathrm{NaCl}$ and 5\% (w/v) SDS to a final protein concentration of $10 \mu \mathrm{M} .10 \mathrm{mM}$ stock solutions of either Alexa Fluor $488 \mathrm{C}_{5}$ maleimide, Alexa Fluor $633 \mathrm{C}_{5}$ maleimide, or Bodipy-FL maleimide (Life Technologies, Grand Island, NY) in DMSO were then diluted 100-fold into the solutions to initiate labeling. Labeling reactions were then covered in argon gas, sealed, and tumbled in the dark at room temperature overnight. SDS was used as a solubilizing detergent for labeling in order to prevent aggregation and to ensure the proteins remained monomeric throughout the course of the reaction. To remove unreacted fluorophore, the samples were then passed through two consecutive $5 \mathrm{~mL} 7 \mathrm{KDa}$ MWCO Zeba desalting columns (Life Technologies, Grand Island, NY), which were pre-equilibrated with 40 mM HEPES ( $\mathrm{pH} 7.5$ ) containing $150 \mathrm{mM} \mathrm{NaCl}$ and 5\% (w/v) SDS according to the manufacturer's instructions.

\section{Reconstitution into Proteoliposomes}

Stock solutions of the lipids 1-palmitoyl-2-oleoyl-sn-glycero-3-phosphocholine (POPC) (Avanti Polar Lipids, Alabaster, AL), N-palmitoyl-D-erythro-sphingosylphosphorylcholine (PSM) (Avanti Polar Lipids, Alabaster, AL), cholesterol (Sigma Aldrich, St. Louis, MO), 1,2-dioleoylsn-glycero-3-phosphoethanolamine-N-(lissamine rhodamine B sulfonyl) (Rh-DOPE) (Avanti

Polar Lipids, Alabaster, AL), and 3,3'-dioctadecyloxacarbocyanine percholorate (DiOC18) (Life Technologies, Grand Island, NY) were produced by suspending powdered lipids in 95:5 EtOH: $\mathrm{H}_{2} \mathrm{O}$. Lipid stocks were dispensed into glass test tubes using a Hamilton syringe (The Hamilton Company, Reno NV) at a molar ratio of 2:2:1 POPC: PSM: Cholesterol. The lipid probes RhDOPE or DiOC18 were then added to a final concentration of $0.1 \mathrm{~mol} \%$ or $0.02 \mathrm{~mol} \%$, 
respectively. A lipid film was then formed by drying the solvent under a stream of nitrogen gas. Residual solvent was then removed by freeze-drying the samples for at least 3 hours. The lipid mixture was then solubilized in mixed micelles by adding $40 \mathrm{mM}$ HEPES (pH 7.5) containing $150 \mathrm{mM} \mathrm{NaCl}, 3.4 \%(\mathrm{w} / \mathrm{v}) \mathrm{SDS}$, and the fluorescently-labeled target protein to the tube and mixing by vortex for 2 minutes under argon gas. The final concentration of lipids and protein were $1.2 \mathrm{mM}$ and $3 \mu \mathrm{M}$, respectively (400:1 molar lipid: protein). Mixed micelles were then equilibrated by tumbling the samples in the dark for at least 1 hour at room temperature.

Rapid removal of solubilizing detergents was previously found to increase the homogeneity of the resulting proteoliposomes (4). To produce homogenous unilamellar proteoliposomes, SDS was rapidly and selectively precipitated using a modified version of a previously reported reconstitution protocol (5). SDS was precipitated by adding the appropriate volume of $4 \mathrm{M} \mathrm{KCl}$ to achieve a 1:1 molar ratio of $\mathrm{K}^{+}$to dodecyl sulfate. The samples were then mixed by vortex for 10 seconds and centrifuged for 15 minutes at $14,000 \mathrm{rpm}$ to pellet the precipitated detergent. The supernatant was then transferred to 12-14 KDa MWCO dialysis tubing (Spectrum Labs, Rancho Dominguez, CA) and dialyzed in the dark against $4 \mathrm{~L}$ of $10 \mathrm{mM}$ HEPES (pH 7.5) in order to remove residual detergent and salt. Dialysis was allowed to proceed for up to two days with regular changes of the bath until no detergent could be detected in the dialysis bath. Proteoliposomes were either stored under argon at $4^{\circ} \mathrm{C}$ in the dark for short term use or flash-frozen for long term storage.

\section{GUV formation and Confocal Fluorescence Microscopy}

GUV's were formed from proteoliposomes using a protocol based on that of Girard et al.(6)

Briefly, an array of $2 \mu \mathrm{L}$ drops of the proteoliposome solutions were deposited on the surface of 
indium tin oxide-coated glass slides and dried under controlled humidity in a desiccator containing a saturated sodium chloride solution for one hour in the dark. A chamber was then formed around the semi-dry proteoliposome films using a rubber O-ring with a thin coating of silicon grease (Corning, Corning, NY). The chamber was then filled with $100 \mathrm{mM}$ sucrose prior to sealing the chamber by applying a second indium tin oxide-coated slide to the top of the Oring. The slides were then clipped together and connected to electrodes in a manner to allow passage of current through the chamber. A wave generator was then used to pass a sinusoidal current through the chamber with a frequency of $10 \mathrm{~Hz}$ at $1 \mathrm{~V}$. Electroswelling of GUVs was allowed to proceed for at least 2 hours in the dark at $50^{\circ} \mathrm{C}$. The sucrose solution containing GUVs was then added dropwise to a glass test tube containing $3 \mathrm{~mL}$ of $100 \mathrm{mM}$ glucose that was pre-warmed to $50^{\circ} \mathrm{C}$ prior to incubation at room temperature for at least 20 minutes in the dark. $100 \mu \mathrm{L}$ of solution containing intact GUVs was then harvested from the bottom of the tube and imaged using a Zeiss 510 scanning laser confocal fluorescence microscope with a 40x 1.4 NA Zeiss Plan-Neofluor objective (Carl Zeiss, San Diego, CA). Samples containing Alexa Fluor 488-labeled proteins or the DiOC18 lipid tracer were excited with an argon laser at $488 \mathrm{~nm}$ and detected with a 505-550 $\mathrm{nm}$ band pass emission filter. Samples containing the rhodamine DOPE lipid tracer were excited with a $546 \mathrm{~nm}$ laser and detected with a long pass $560 \mathrm{~nm}$ emission filter. Samples containing Alexa Fluor 633-labelled proteins were excited with a $633 \mathrm{~nm}$ laser and detected using a long pass $650 \mathrm{~nm}$ emission filter. Phase separation was apparent in the vast majority of GUVs produced. Only images in which the protein signal was clearly detectable were counted in the analysis. 


\section{Supplemental References}

1. Kim, J. H., D. Peng, J. P. Schlebach, A. Hadziselimovic, and C. R. Sanders. 2014. Modest effects of lipid modifications on the structure of caveolin-3. Biochemistry 53:4320-4322.

2. Barrett, P. J., Y. Song, W. D. Van Horn, E. J. Hustedt, J. M. Schafer, A. Hadziselimovic, A. J. Beel, and C. R. Sanders. 2012. The amyloid precursor protein has a flexible transmembrane domain and binds cholesterol. Science 336:1168-1171.

3. Schlebach, J. P., D. Peng, B. M. Kroncke, K. F. Mittendorf, M. Narayan, B. D. Carter, and C. R. Sanders. 2013. Reversible folding of human peripheral myelin protein 22, a tetraspan membrane protein. Biochemistry 52:3229-3241.

4. Levy, D., A. Gulik, A. Bluzat, and J. L. Rigaud. 1992. Reconstitution of the sarcoplasmic reticulum $\mathrm{Ca}(2+)$-ATPase: mechanisms of membrane protein insertion into liposomes during reconstitution procedures involving the use of detergents. Biochim Biophys Acta 1107:283-298.

5. Popot, J. L., J. Trewhella, and D. M. Engelman. 1986. Reformation of crystalline purple membrane from purified bacteriorhodopsin fragments. EMBO J 5:3039-3044.

6. Girard, P., J. Pecreaux, G. Lenoir, P. Falson, J. L. Rigaud, and P. Bassereau. 2004. A new method for the reconstitution of membrane proteins into giant unilamellar vesicles. Biophys J 87:419429. 


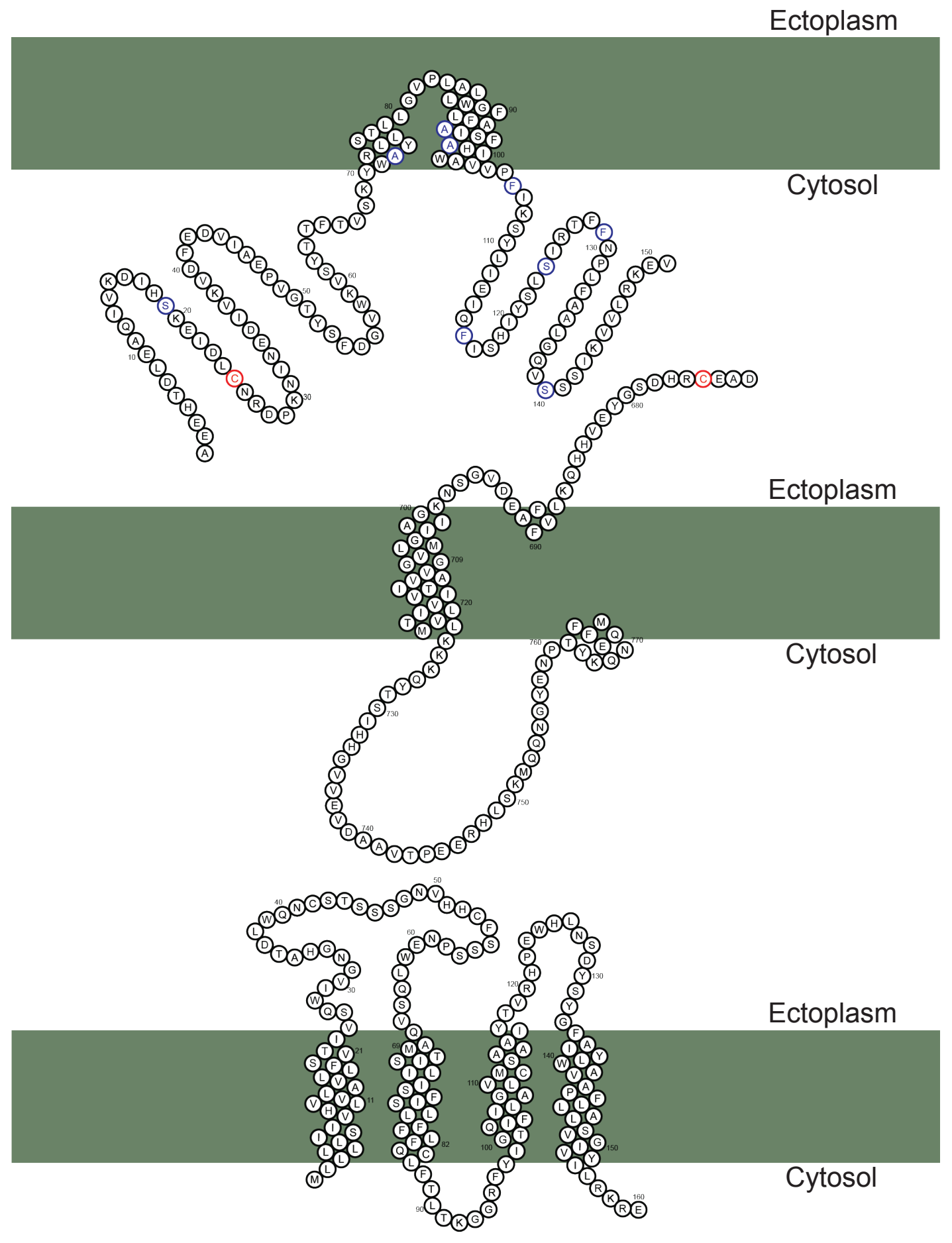

Supplementary Figure 1. Topology of Cav3, C99, and PMP22. Cartoons depict the topology of Cav3 (top), C99 (middle), and PMP22 (bottom) with respect to the membrane (gray). Mutated native cysteines are shown in blue. Non-native cysteines introduced for fluorescent labeling are shown in red. Fluorophores were conjugated to PMP22 using its four native cysteines, which include residues 42, 53, 85, and 109 . 
RhDOPE

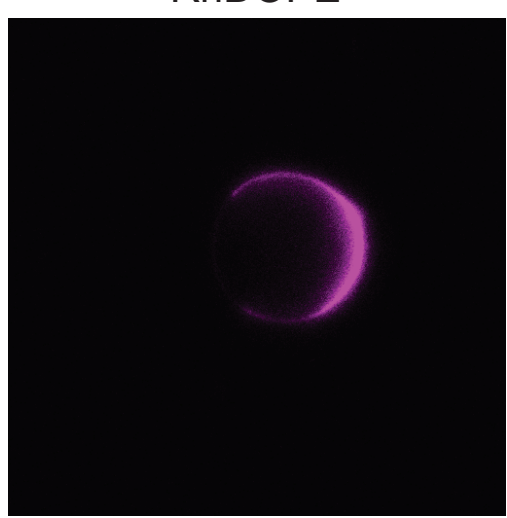

BodipyFL

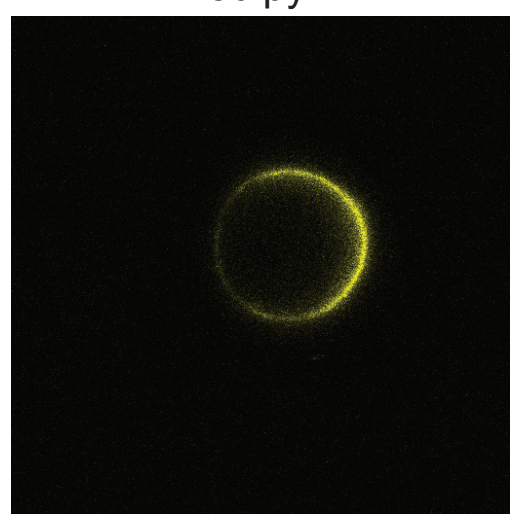

Merge

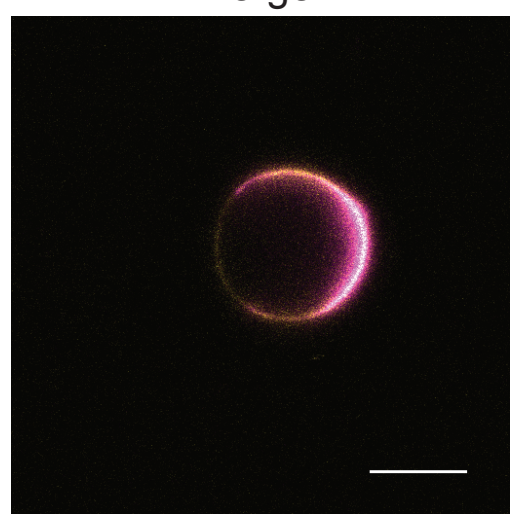

Supplementary Figure 2. Partitioning of bodipy FL-Caveolin 3 in phase-separated GUVs. Bodipy FL-labeled A72C Cav3 was incorporated into SDS mixed micelles containing a 2:2:1 molar ratio of POPC: PSM: Cholesterol with $0.1 \mathrm{~mol} \%$ of the fluorescent tracer lipid rhodamine DOPE at a bulk molar lipid: protein ratio of 400:1. To form proteoliposomes, SDS was removed by dialyzing against $4 \mathrm{~L}$ of $10 \mathrm{mM} \mathrm{(2:1)} \mathrm{Tris/} \mathrm{MES} \mathrm{buffer} \mathrm{(pH} \mathrm{7.0).} \mathrm{Proteoliposomes} \mathrm{were} \mathrm{then}$ subjected to three freeze-thaw cycles to ensure the vesicles were unilamellar. GUVs were then formed from proteoliposomes and imaged as described in Supplemental Methods. A representative image of a GUV containing Bodipy FL-labeled caveolin-3 is shown along with a $10 \mu \mathrm{m}$ scale bar for reference. 

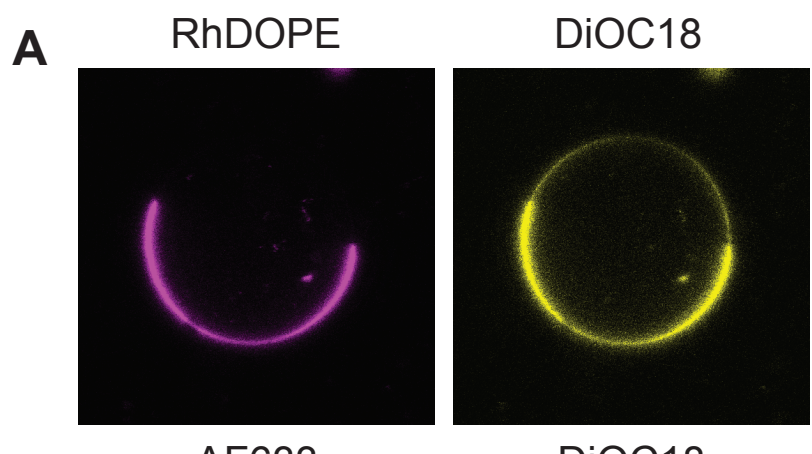

DiOC18

B

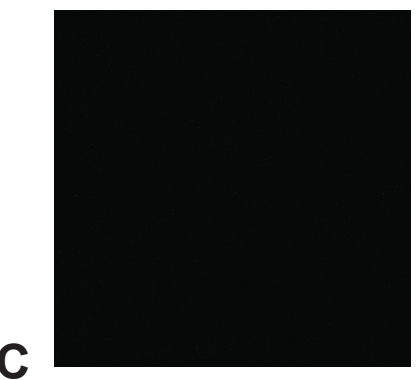

C

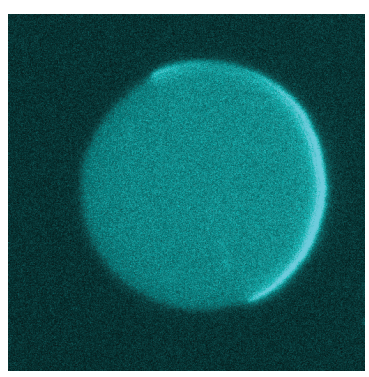

D

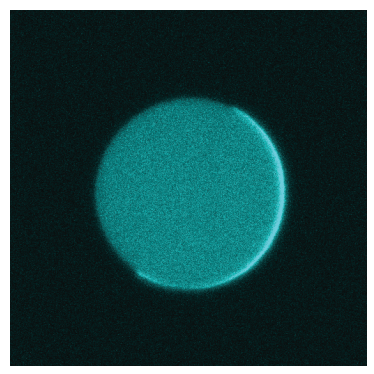

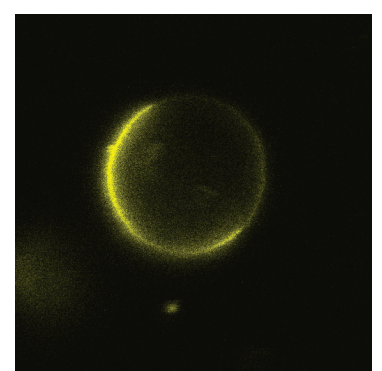
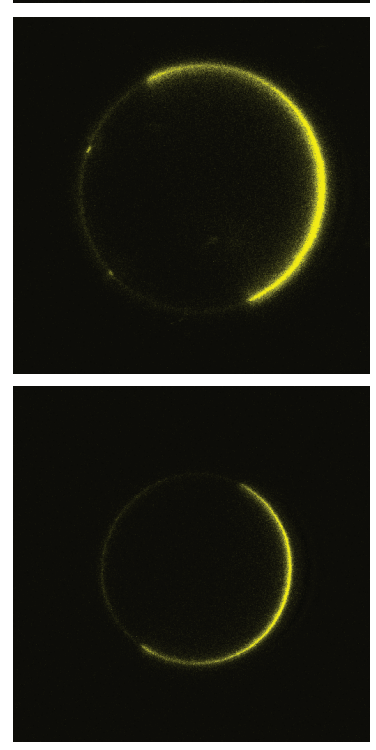

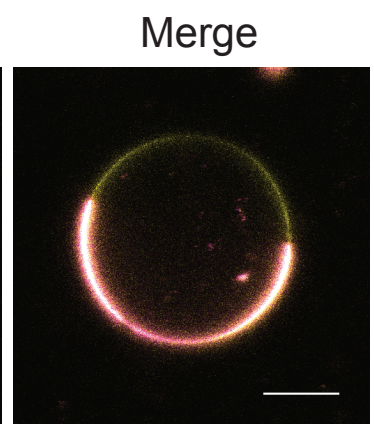

Merge
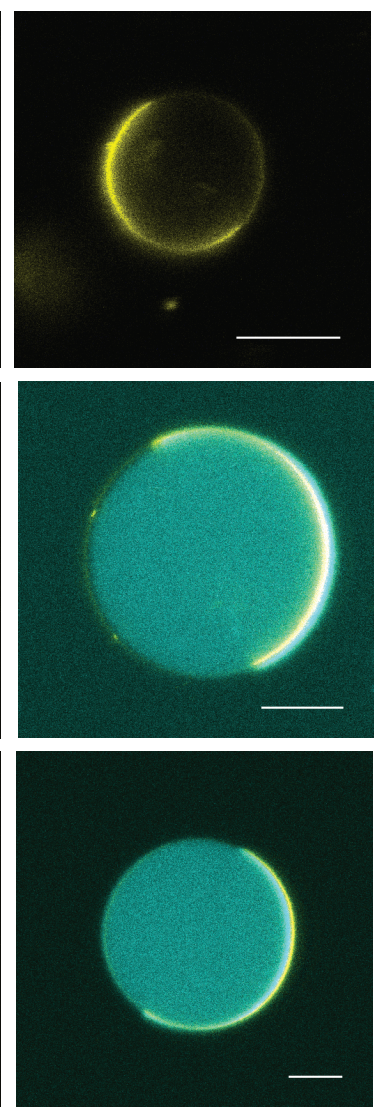

Supplementary Figure 3. Partitioning of Alexa Fluor 633-labeled C99 and PMP22 in phaseseparated GUVs marked with DiOC18. GUVs composed of 2:2:1 POPC: PSM: Cholesterol and various lipid probes and proteins were formed and imaged as is described in Materials and Methods. A) A representative image of GUVs containing 0.1 mol\% Rhodamine DOPE and 0.02 mol\% DiOC18 lipid tracers is shown. The overlap of the probes demonstrates that DiOC18 is primarily resides within the $\mathrm{L}_{\mathrm{d}}$ phase. B) A representative image of GUVs containing $0.02 \mathrm{~mol} \%$ DiOC18 demonstrates a lack of spill-over into the protein channel. C) A representative image of GUVs containing DiOC18 and Alexa Fluor 633-C99 is shown. The overlap between DiOC18 and C99 demonstrates that $\mathrm{C} 99$ resides within the $\mathrm{L}_{\mathrm{d}}$ phase under this condition. D) A representative image of GUVs containing DiOC18 and Alexa Fluor 633-PMP22 is shown. The overlap between DiOC18 and PMP22 demonstrates that PMP22 resides within the $\mathrm{L}_{\mathrm{d}}$ phase under this condition. $10 \mu \mathrm{m}$ scale bars (white) are shown for reference. 